Service social

\title{
Femmes immigrantes et travail social : une recension des écrits
}

\section{Gisèle Legault et Elda Rojas-Benavente}

Volume 37, numéro 1-2, 1988

Par-delà les barrières des sexes

URI : https://id.erudit.org/iderudit/706391ar

DOI : https://doi.org/10.7202/706391ar

Aller au sommaire du numéro

Éditeur(s)

École de service social de l'Université Laval

ISSN

1708-1734 (numérique)

Découvrir la revue

Citer cet article

Legault, G. \& Rojas-Benavente, E. (1988). Femmes immigrantes et travail social : une recension des écrits. Service social, 37(1-2), 176-196.

https://doi.org/10.7202/706391ar

\section{Résumé de l'article}

La clientèle des femmes immigrantes est relativement peu connue en service social. Cet article, amorce d'une recherche sur la pratique sociale auprès de ces femmes, constitue une recension d'une soixantaine d'écrits sur le sujet, en service social, psychologie, psychiatrie, sociologie, anthropologie, et parus au cours des dix dernières années. Il couvre les volets suivants : concepts théoriques de féminité et d'ethnicité et leur mise en relation ; travail salarié des femmes immigrantes : principales caractéristiques et mesures pouvant améliorer leur situation ; immigration et rôles sociaux, avec emphase sur le partage des tâches, les rapports de pouvoir dans la famille et la place du réseau de parenté ; santé des femmes immigrantes : leurs préoccupations, leurs expériences avec le système de soins, leurs attitudes vis-à-vis certains malaises, l'impact du choc culturel sur leur santé; le counseling interculturel : son développement et sa définition à partir des écrits en service social principalement. 
LEGAULT, Gisèle, professeure à l'École de service social de l'Université de Montréal.

ROJAS-BENAVENTE, Elda, étudiante à la maîtrise en service social à l'Université de Montréal.

\section{Femmes immigrantes et travail social : une recension des écrits}

\section{Gisèle Legault Elda Rojas-Benavente}

En service social, la clientèle des femmes immigrantes est relativement peu connue. Ayant préparé une rencontre sur le sujet, à l'intérieur d'un cours sur la condition féminine au début des années 1980, nous avions trouvé relativement peu d'écrits et de documents. L'essentiel de la rencontre avait été sous forme d'échange avec une travailleuse sociale du Centro Donne, centre de femmes italiennes du quartier SaintMichel, à Montréal. Depuis, dans le milieu francophone de Montréal, la pratique sociale auprès des minorités culturelles s'est précisée, et nous estimons qu'il est important d'en spécifier les modalités particulières. Cet article se veut une contribution dans ce sens. En mars 1988, nous entreprenions une recherche sur la pratique sociale auprès des femmes immigrantes, dans le but d'apprécier l'adéquation ou non des services sociaux par rapport aux attentes et besoins de cette clientèle spécifique.

Dans un premier temps, nous avons recensé les écrits des revues et périodiques ${ }^{1}$ pertinents à notre sujet. II nous importait de connaître les publications existantes, les recherches entreprises, de même que les expériences tentées ailleurs, avant d'entreprendre une étude-terrain de l'expérience québécoise dans le domaine. Nous avons ainsi fouillé tous les numéros des revues et périodiques parus au cours des dix dernières années à la recherche d'écrits portant globalement sur la pratique sociale auprès des minorités culturelles. Il est ressorti de cette opération un nombre considérable d'articles se rapportant de près ou de loin à notre sujet. Nous avons donc divisé notre sujet principal en sous-sujets et constitué des bibliographies correspondantes. Pour le S.S., vol. 37, nos 1 et $2,1988$. 
présent article, nous avons retenu les items se rapportant essentiellement aux femmes immigrantes. La bibliographie correspondante se trouve en fin d'article. D'autres bibliographies ont été constituées pour les autres sous-sujets et portent sur la pratique sociale auprès des minorités culturelles ( 50 articles), la pratique sociale auprès des réfugiés ( 13 articles), l'éducation en service social et la pratique interculturelle (12 articles), les concepts théoriques d'ethnicité et de minorité (17 articles).

Nous avons également utilisé une bibliographie déjà constituée par Joyce Scane sur le sujet, et présentée dans le numéro de mars 1987 de la revue Ressources for Feminist Research ${ }^{2}$. Des 81 rubriques composant cette bibliographie, 43 ont été retenues : plusieurs constituaient des instruments d'intervention plutôt que des articles ou résultats de recherches ; certaines autres, venant de petits groupes communautaires, n'ont pu être retracées.

Afin de compléter la recension des écrits, nous avons effectué une recherche bibliographique par ordinateur, à partir de mots-clés ${ }^{3}$ soumis à trois banques de données, soit Social Work Abstracts, Sociological Abstracts et Psychological Abstracts, qui ont fourni respectivement 21, 56 et 26 références, dont dix-huit ont été conservées en raison de leur pertinence pour le sujet à l'étude. Certaines de ces références avaient déjà été répertoriées dans la première étape de la recherche ; d'autres, après lecture des résumés fournis par les banques de données, ne présentaient pas d'intérêt pour notre étude.

Les articles portant sur les femmes immigrantes couvraient plusieurs sujets que nous avons délimités de la façon suivante : concepts théoriques d'ethnicité et de féminité, femmes immigrantes et travail salarié, immigration versus rôles sociaux, femmes immigrantes et santé, counseling interculturel.

\section{Concepts d'ethnicité et de féminité}

Pour aborder ces concepts, nous nous référons essentiellement à Juteau-Lee et Roberts (1981) et $\mathrm{Ng}(1981,1986)$. Peu d'écrits mettent en relation ethnicité (ou minorité ethnique) et féminité, aussi avons-nous pensé que l'articulation de ces concepts, par les auteures mentionnées, aiderait à la compréhension de la problématique des femmes immigrantes.

Disons, d'entrée de jeu, que l'ethnicité et la féminité sont présentées comme des construits sociaux et non comme des données "naturelles" ou innées. Alors que naître du sexe féminin est une donnée de la nature qui inclut, entre autres, l'habileté de porter des enfants et de les allaiter, 
être féminine renvoie à un apprentissage social dont les modalités ont été maintes fois soulignées dans les études féministes, et plus près de nous, celle de Simard ${ }^{4}$. Ce qui était vu comme naturel, immuable, est maintenant perçu comme socialement construit. On considérera ainsi comme féminines les personnes ayant certains traits de caractère (soumission, passivité, dépendance) et certaines caractéristiques comportementales dont la plus importante est celle d'être affectées aux dimensions "naturelles" et "biologiques» de l'expérience humaine (Guillaumain, 1978). Cette pré-détermination prive les femmes des statuts d'adulte et d'humain à part entière, comme l'ont démontré les travaux de Broverman, De Beauvoir et Millet ${ }^{5}$. À cette position de minoritaires correspond une expérience de subordination. La féminité devient alors une caractéristique d'un groupe subalterne. Un parallèle peut être tracé entre les femmes et les groupes ethniques: selon les perspectives présentées par JuteauLee et Roberts, féminité et ethnicité sont apprises et constituent des construits sociaux qui correspondent à des situations socio-économicopolitiques dans lesquelles femmes et groupes ethniques ne sont pas nés, mais sont placés.

L'ethnicité, tout comme la féminité, requiert un apprentissage élaboré d'actions et de pratiques qui reflètent l'appartenance et parmi lesquelles nous retrouvons la langue, certains mouvements corporels, certains types de comportements, plus particulièrement la soumission au groupe dominant et l'acceptation de certains travaux considérés « appropriés " pour les membres d'un groupe ethnique. L'ethnicité et la féminité, en tant que significations sociales, sont ensuite utilisées comme explications des attitudes et gestes des individus. Comme le mentionne Roberts $^{6}$, "les Italiens/les femmes sont comme ceci, traditionnels/ convervatrices, ce sont leurs valeurs, ils/elles n'ont pas les mêmes priorités que les Anglais/hommes, leur apparence est différente, ils/ elles sont plus centrés sur la famille, ils/elles aiment, sont meilleurs(es) aux besognes sales et mal payées ». En tant que construits sociaux, l'ethnicité et la féminité sont utilisées pour diriger des personnes vers des catégories de travail rattachées à certains types de reconnaissance sociale ou pour les y maintenir. Selon cette analyse, la féminité et l'ethnicité sont des systèmes spécifiques de subordination, l'un aux hommes, l'autre aux non-ethniques ou à la culture dominante. Ethnicité et féminité conjugées créent un phénomène d'isolement, de solitude, souvent d'impuissance, dont les études sur les femmes immigrantes font état plus loin.

Une autre auteure $(\mathrm{Ng}, 1986)$ s'inscrit dans la même ligne de pensée en démontrant que l'ethnicité des femmes immigrantes ne devient apparente qu'à des échelons précis de la hiérarchie occupationnelle canadienne. C'est ainsi que certaines personnes seront socialement 
considérées immigrantes ou ethniques alors que d'autres, non, les unes et les autres étant pourtant également étrangères. L'ethnicité, qui n'existe pas en soi mais est socialement produite, ne devient un facteur déterminant de l'expérience des femmes que lorsque celles-ci occupent des emplois réservés à la main-d'œuvre féminine étrangère (Labelle et al, 1987). Ng en est venu à ces conclusions après avoir observé le processus de counseling d'emploi d'un bureau fédéral, à Vancouver, s'occupant spécifiquement des femmes étrangères ne parlant pas l'anglais. Elle a ainsi observé que le conseiller sélectionnait, à même l'histoire de vie et de travail de la femme immigrante, les habiletés et expériences pouvant être traduites en compétences manquantes et souhaitées sur le marché du travail canadien. Son rôle en était un de juxtaposition des capacités des femmes aux impératifs du marché du travail canadien, en fonction de certains types d'emploi difficilement assurés par la maind'œuvre locale, à savoir ceux du secteur secondaire, tels que décrits par Hélène David 7 , et largement occupés par les femmes, y compris les immigrantes. C'est ce qui fait dire à $\mathrm{Ng}$ (1981) que la situation et l'expérience des immigrantes sont révélatrices de l'organisation de la société canadienne, où ces femmes sont identifiées comme " ethniques " du moment qu'elles s'insèrent dans une stratification sociale prévoyant leur intégration à des échelons spécifiques, de façon à répondre aux mécanismes de notre marché du travail.

\section{Femmes immigrantes et travail salarié}

L'une des données fondamentales de la situation des femmes immigrantes est leur insertion en grand nombre sur le marché du travail. Labelle et Kempeneers (1988) sont explicites à ce sujet. Ces femmes, qui représentent près de la moitié de la population étrangère au Québec, sont une composante importante des migrations de travail. Les sources d'immigration se sont toutefois considérablement modifiées depuis les années soixante. Alors que l'immigration féminine provenait essentiellement du Royaume-Uni $(22,9 \%)$, des États-Unis $(13,3 \%)$ et de l'Europe $(58,2 \%)$, les femmes des Caraïbes, de l'Asie et de l'Afrique prennent une importance accrue parmi les principaux groupes d'immigrées, au cours des années 1970. La situation de crise économique et politique dans plusieurs pays du Tiers Monde, provoque également un exode de réfugiés qui ira en augmentant au cours des années 1970 et 1980. Les mêmes tendances peuvent être observées au Canada (Seward et McDade, 1988). Ces changements dans la provenance des populations 
immigrées, répondent, entre autres, à des modifications de la structure du marché du travail chez nous et à des besoins nouveaux en maind'œuvre internationale, particulièrement au chapitre de l'immigration de travailleuses non qualifiées.

En 1981, les femmes constituent $38 \%$ de la main-d'œuvre immigrée, $9 \%$ des travailleuses québécoises et $4 \%$ de la main-d'œurre totale. Leur insertion sur le marché du travail québécois se fait toutefois selon des paramètres précis visant à les concentrer dans un nombre restreint de secteurs économiques et dans les emplois souvent désertés par les populations locales. À un pôle se situe la fraction racialement/ethniquement et sexuellement catégorisée que constituent les travailleuses de manufacture, des services personnels, de l'entretien ménager industriel, de l'hôtellerie, etc. Ces femmes proviennent d'Europe du Sud et du Tiers Monde. C'est le lieu des postes manuels et d'exécutants, mal payés, souvent non syndiqués, précaires, n'offrant aucune voie de promotion, peu ou pas d'avantages sociaux, présentant une forte discontinuité et un taux élevé de rotation dans l'emploi (Labelle et al., 1987). Une autre auteure (Rytina, 1981) en arrive à la conclusion dans son étude que la participation désavantageuse des immigrantes est largement due au fait que la migration est souvent décidée en fonction des possibilités de travail du mari et que cet événement coïncide avec la période où les femmes ont et élèvent leurs jeunes enfants, et souvent sans aide de leur conjoint.

À l'autre pôle se situe une faible proportion d'immigrées plus qualifées, œuvrant dans les secteurs de l'enseignement, des services de santé et des services sociaux ou de l'administration. Venant du RoyaumeUni, de France, des États-Unis ou du Tiers Monde, elles sont arrivées avant 1961, dans le contexte de prospérité économique de la Révolution tranquille.

Une étude canadienne récente (Seward et McDade, 1988) nous révèle que les immigrantes semblent se porter mieux économiquement que les femmes nées au Canada, leur revenu moyen en 1980 était en effet de $9 \%$ supérieur à celui des canadiennes nées ici. Les auteures ajoutent toutefois que ces données, à prime abord surprenantes, dissimulent la forte hétérogénéité du groupe des immigrantes en fonction de leur pays d'origine et de la durée de leur séjour au Canada. De plus, elles précisent qu'un grand nombre de ces femmes restent encore cloisonnées dans les emplois sous-payés.

Cette étude est particulièrement intéressante en ce qu'elle propose des mesures pouvant améliorer la situation de travail des femmes immigrantes au chapitre des catégories d'immigration, de la formation linguistique et professionnelle, de la reconnaissance des acquis et de la garde des enfants. Au chapitre des catégories d'immigration, les femmes 
immigrantes appartiennent majoritairement à la catégorie familles (les deux autres étant les catégories réfugiés et immigrants indépendants), ce qui fait d'elles des citoyennes «parrainées", habituellement par leur conjoint. Cette catégorisation les limite sérieusement dans l'accès à la formation linguistique, à l'aide sociale, à l'aide juridique et aux logements subventionnés par les pouvoirs publics.

Au chapitre de la formation linguistique, Paredes (1987) mentionne plusieurs cas d'exclusion des femmes immigrantes à Toronto : celles qui ne se destinent pas au marché du travail, celles qui seront orientées vers des emplois non qualifiés requérant peu l'usage de l'anglais, celles qui sont parrainées. Elle attire aussi l'attention sur l'organisation de cette formation, à savoir à temps plein, le jour, pour une durée maximum de six mois et exigeant du travail personnel d'intégration le soir, alors que la femme est fatiguée et doit aussi s'occuper de ses enfants et de la maison. Paredes reproche aux programmes de formation linguistique de ne pas faire appel à l'expérience spécifique des femmes et de ne pas tenir compte de leurs handicaps au chapitre des habitudes d'apprentissage. Par ailleurs, elle mentionne l'existence de certains programmes mis sur pied par des groupes communautaires en fonction des intérêts et expériences des femmes immigrantes. Pour leur part, Seward et McDade recommandent une plus large admissibilité des femmes à la formation linguistique, de façon à ce qu'elles ne constituent plus les $61 \%$ des personnes ne parlant ni l'une ni l'autre des langues officielles au Canada, facteurs qui les handicapent sérieusement sur le marché du travail, sans oublier l'isolement et la dépendance dans lesquels cette réalité les place.

Au chapitre formation et perfectionnement, les auteurs soulignent que les immigrantes bénéficient rarement de l'un ou l'autre des programmes fédéraux suivants : intégration professionnelle, acquisition de compétence, développement de l'emploi, pénurie de main-d'œuvre, développement des collectivités et aide à l'innovation. Il semble que leur choix d'emploi et leur renonciation aux cours soient dictés par leurs responsabilités familiales et l'absence de garderies abordables, accessibles, et qui tiennent compte des particularités culturelles. Concernant la formation, prise dans son sens le plus large cette fois, un auteur français (Barbé, 1978) nous met en garde contre des programmes trop traditionnellement féminins tels que cours de couture, cuisine, économie familiale ou des formations trop uniquement socio-éducatives telles que poterie ou tissage ; l'auteur nous rappelle aussi l'importance d'évaluer avant tout les besoins des femmes en partant de leur trajectoire antécédente et d'adopter une méthodologie de formation qui permette une progression : de l'objet à connaître à la conceptualisation, puis à l'utilisation du même objet. 
Steward et McDade soulignent également la difficulté de reconnaître les acquis ou d'en établir les équivalences, surtout si les connaissances des immigrantes ont été acquises ailleurs qu'en Europe et en Amérique du Nord. Ceci constitue un handicap additionnel au chapitre du travail salarié et contribue à leur rendre difficile l'accès des emplois correspondant à leurs compétences.

Concernant la garde des enfants, les auteures mentionnent que les ressources sont souvent inexistantes ou inabordables, contraignant les femmes immigrantes à du travail à domicile pour lequel le salaire est médiocre, les journées longues et les conditions de travail détestables. Lorsqu'ils leur sont accessibles, les programmes de garde d'enfants reflètent alors la culture dominante: dans le choix des jeux, des mots, des activités, des contes. La disponibilité de services de garde d'enfants est d'autre part un facteur déterminant de la participation des immigrantes au marché du travail, du type d'emploi qu'elles acceptent et de leur capacité à profiter des programmes de formation.

\section{Immigration et rôles sociaux}

Lovel et al. (1987) attirent notre attention sur les changements dans les rôles sociaux auxquels les femmes ont à faire face lorsqu'elles émigrent et qui sont plus considérables que pour les hommes. Ces changements sont dûs principalement au fait qu'elles doivent alors conjuguer travail salarié et vie de famille, ce qui n'était pas toujours le cas ou se faisait plus facilement dans le pays d'origine. Aussi, trouvonsnous très appropriée la question posée par Labelle et al. (1987) : "S'agitil d'une accession à la modernité (par le transfert dans une société plus ouverte) ou d'un processus d'émancipation (la capacité pour la femme de gagner de l'argent remettant en cause son rôle subordonné) ou n'assiste-t-on pas plutôt au renforcement des rôles traditionnels et à l'accroissement du pouvoir des hommes du fait de la migration ? 8

Les mêmes auteures nous apportent des éléments de réponse en ce qui a trait au partage des rôles et aux rapports de pouvoir au sein du ménage. Des soixante-seize femmes immigrées interviewées, le tiers seulement ont pu compter sur leur conjoint pour les aider, et le plus souvent, il s'agit d'une aide ponctuelle et circonscrite. Dès que la femme a un travail (de couture) à la maison ou qu'une autre femme demeure avec la famille (souvent la mère de l'époux) ou que l'une des filles est assez vieille, le conjoint se désengage. En somme, on n'est pas loin du modèle qui prévalait au pays d'origine; s'il y a apparition de 
nouveaux modèles, c'est plutôt dans ceux que la femme essaie d'inculquer à ses enfants.

En ce qui a trait aux rapports de pouvoir au sein du ménage, ces chercheuses ont constaté que pour la femme immigrante, le fait de gagner de l'argent ne changeait pas le rapport de pouvoir au sein du couple, l'homme y demeure l'autorité. Le travail salarié de la femme est vu comme un travail d'appoint, un supplément au budget familial, celle-ci en retire peu de valorisation sociale (Bonato, 1983). Ainsi l'étude de Labelle et al. rapporte que les items budgétaires sont ainsi répartis entre les conjoints : alors que l'homme s'occupe des dépenses importantes (logement, appareils), la femme prend charge des dépenses "élastiques " ou périssables (habillement, nourriture), ce qui crée un rapport de pouvoir inchangé au sein du couple. Les chercheuses ajoutent que I'homme, qu'il soit conjoint, père ou frère, continue d'exercer un certain contrôle sur les faits et gestes de la femme, plus particulièrement sur ses sorties, ses fréquentations et son comportement public. Dans certains cas, le conjoint utilise la violence physique pour renforcer son pouvoir. Lovel et al. (1987) font part à cet effet d'une intervention auprès des femmes du Sud-est Asiatique, victimes de violence conjugale, et insistent sur la nécessité de s'adjoindre des personnes du milieu qui puissent servir de modèle d'ajustement bi-culturel à la nouvelle société, en l'occurrence celle de Seattle aux États-Unis.

Si travail salarié et vie de famille se conjuguaient plus facilement pour les femmes, dans leur pays d'origine, c'est que le réseau de parenté y jouait un rôle différent. Dans l'étude de Labelle et al., on note une diminution, dans le pays d'accueil, des ménages composés de familles étendues (de $50 \%$ dans le pays d'origine, on passe à $20 \%$ au Québec) et conséquemment, une réduction de l'aide concrète reçue de cette façon. Parallèlement, les familles nucléaires et monoparentales augmentent. On note toutefois qu'alors que les réseaux d'entraide traditionnels tendent à se relâcher, ils se reconstituent à l'échelle internationale, sous diverses formes : contacts par lettres, téléphones, visites, partage de renseignements pratiques, envoi d'aide matérielle, échange de services, surtout au chapitre des tâches dites féminines (garde et soins aux enfants).

\section{Femmes immigrantes et santé}

Plusieurs écrits s'attardent à la santé des femmes immigrantes, à leurs préoccupations en ce domaine, à leurs expériences avec le système 
médical, à leurs attitudes vis-à-vis ce système et ses agents, au fait qu'elles y vivent parfois de la discrimination.

Ainsi Anderson (1985) a interviewé six Indiennes (de l'Inde) et huit Grecques émigrées en Colombie-Britannique et clientes des services de santé de Vancouver. Toutes avaient vécu une transition de la vie rurale à la vie urbaine en émigrant, toutes étaient impliquées dans un travail salarié non spécialisé, principalement du secteur des services, et avaient à conjuguer travail domestique et travail salarié. Ces femmes ont des rapports fréquents avec le système de santé, principalement avec les infirmières, mais ce qui les préoccupe davantage, ce sont des sentiments de solitude, de tension et de dépression, un sentiment global d'être " marginales ». Les deux groupes de femmes n'ont toutefois pas la même expérience dans leurs contacts avec le système de santé. Alors que les Grecques s'y sentent comprises et y abordent l'ensemble de leurs préoccupations, il n'en est pas de même des Indiennes qui, outre des problèmes de langue plus considérables, ne se sentent pas capables d'exprimer leurs difficultés de façon satisfaisante. L'auteure attribue principalement cette différence à des facteurs socio-économicopolitiques particuliers qui affectent les deux communautés, au Canada, où les Indiennes y sont davantage victimes de discrimination à cause peut-être de leur plus grande "visibilité ". L'auteure conclut en recommandant une meilleure formation des intervenants(es), surtout en ce qui a trait à l'appréciation des contextes socio-culturels et politiques d'où ces symptômes émergent, de façon à éviter, comme ce fut le cas pour les Indiennes, des conseils ou suggestions non appropriés à ces groupes. Elle recommande aussi une décentralisation des services de santé et un rapprochement des groupes communautaires, davantage informés et conscients des particularités culturelles des milieux qu'ils desservent.

Une autre auteure (Lewin, 1979) souligne la place qu'occupent la maladie et la consultation médicale dans l'univers de l'immigrante, plus particulièrement la femme latino-américaine émigrée sur la côte ouest américaine. Son article intitulé "La noblesse de la souffrance " et rédigé à la suite d'une expérience d'intervention, nous informe que la maladie est en quelque sorte un "congé", pour la femme, de l'altruisme habituellement attendu d'elle, tout en attestant de son sacrifice passé. Sa maladie devient la preuve qu'elle s'est sacrifiée pour le bien de ses enfants et a été une mère vertueuse au point d'en devenir une « martyre ». La maladie lui accorde un répit des pressions de son rôle de mère "altruiste et oublieuse d'elle-même ". La caution de la maladie l'autorise alors à s'occuper d'elle, à prendre congé des rôles domestiques et maternels et à se permettre ce loisir qu'est alors une visite au centre de soins et la rencontre d'autres femmes dans la même situation. Les 
femmes latino-américaines expriment aussi, à travers leur maladie, les insatisfactions reliées à leur situation de pauvreté et d'émigration. L'aspect temporaire et guérissable de leurs maladies, souligne l'auteure, est toutefois important, sans quoi elles perdraient beaucoup de leur raison d'être.

Un phénomène semblable est investigué par Wolf-Dresp (1985) au Massachussetts, à partir d'interviews avec des femmes porto-ricaines souffrant de " nervios ", trouble fréquemment mentionné en consultation par les femmes latino-américaines vivant aux États-Unis. Ce syndrôme, causé par une perte, une maladie, des relations conflictuelles à l'intérieur de la famille ou avec la société en général, se manifeste par un état d'agitation et d'anxiété avec composantes somatiques, des changements d'humeur ou d'affect ou encore, des changements d'habitudes alimentaires. Le terme «nervios » semble référer à des émotions et des conflits vécus d'une manière spécifique et propres à la communauté hispanophone. Il est frappant de constater, entre autres, que tous ces symptômes sont tournés vers la personne elle-même, aucun n'exprime la déception ou la colère à l'endroit de causes extérieures pourtant souvent à l'origine des malaises.

Souffrant de "nervios", la femme porto-ricaine souhaite support, aide et compréhension de sa famille ou de sa parenté. Ce soutien ne peut toutefois lui être apporté, en raison des changements socioculturels et politiques auxquels la famille a eu à s'adapter et des nombreux facteurs de stress qui l'ont affectée. Plusieurs trouvent un soulagement dans des activités calmantes telles que l'activité physique et les techniques respiratoires. La consommation de thés médicinaux est aussi fréquente. Plusieurs consultent toutefois les professionnels de la santé qui leur dispensent surtout une aide médicamenteuse dont elles sont insatisfaites. Wolf-Dresp, comme Anderson, conclut en mettant l'accent sur les implications de son étude pour l'intervention auprès des femmes hispanophones. Elle souligne ainsi l'importance, pour l'intervenant(e), de saisir les messages cachés derrière les symptômes de " nervios » et d'aider les femmes à rendre explicites leurs insatisfactions. Les sentiments d'impuissance, de frustration et possiblement de colère deviennent ainsi connectés aux facteurs de stress ; des solutions peuvent alors être envisagées. Elle recommande avant tout un travail visant le renforcement personnel de la femme immigrante qui, en consultant, exprime souvent un malaise familial, à la façon d'un individu qui, par ses symptômes, met à jour un malaise familial, tel que souvent démontré en thérapie familiale. Ce travail de renforcement devrait inclure une révision des rôles traditionnels dans la famille porto-ricaine, entre autres l'identification quasi totale de la femme à son rôle de mère. Ce 
travail encourage également un examen des possibilités d'amélioration de sa situation économique, à travers le travail rémunéré par exemple.

Les sentiments d'inquiétude, de solitude, d'isolement, de dépression reviennent souvent chez les femmes immigrantes, lorsqu'on s'attarde à leur santé physique et mentale. Ainsi, suite à l'observation des contenus thématiques d'un groupe d'intervention auprès de porto-ricaines déprimées, Comas-Diaz (1984) nous fait part de ses constatations. Cette dernière explique que la femme porto-ricaine se conforme au stéréotype féminin en général et au stéréotype culturel en ce qui a trait à son rôle, faisant d'elle une personne dépendante, non affirmative, soumise, ce qui contribue à sa dépression. Par ailleurs, les facteurs qui affectent l'ensemble des Porto-ricains aux États-Unis tels la pauvreté, les problèmes linguistiques et les barrières culturelles, influencent aussi sa santé générale. En raison de la rupture des routines culturelles telles que façons de s'alimenter, de se vêtir, de se comporter en société, et des attaques à leurs valeurs par la culture dominante, l'auteure estime que l'identité de ces femmes est menacée et que cela affecte leur santé mentale. Elle a donc mené une expérience d'intervention de groupe avec huit immigrantes, sur la côte est, et a traité leur dépression par la restructuration cognitive, méthode basée sur une analyse behaviorale de la dépression. Toutes ces femmes étaient chefs de familles monoparentales vivant de l'assistance de l'État et ne parlant qu'espagnol. Voici les sujets abordés dans ce groupe : symptômes physiques et mentaux, problèmes pratiques, traitement, famille d'origine, conjoint, enfant, relations interpersonnelles, expériences d'enfance et choc culturel. Certaines valeurs culturelles expliquent le choix de sujets plus fréquemment choisis. Ainsi, l'importance des relations personnelles dans la culture porto-ricaine, de même que le concept de "personalismo" rapporté par l'auteure expliquent bien l'emphase mise sur la qualité personnelle de toute transaction ou interaction. De même le thème " enfants " comme sujet de discussion peut être relié à la valeur culturelle de "marianismo". Ce concept basé sur le culte à la Vierge Marie, implique que les femmes doivent d'abord et avant tout être des mères, se sacrifier pour leurs enfants, leur accorder la première place dans leur vie, et sous-entend que la fonction maternelle est la plus noble et qu'elle se rapproche de Dieu.

L'intervention de groupe a été bénéfique à ces femmes. Elles y ont abordé plus facilement leurs sentiments envers les proches, sujet qui, culturellement, ne peut être exprimé directement en consultation. Le thème le plus important et le plus fréquemment abordé est sans contredit le choc culturel défini par l'auteure comme un ensemble de réactions émotionnelles incluant des sentiments tels que inutilité, impuissance, rejet, mésestime de soi, suite à la perte de la famille, des amis, de 
la langue, de valeurs et d'attitudes culturelles. Les renforcements de sa propre culture disparaissent pour l'individu alors que de nouveaux stimulus culturels n'ont pas encore de signification particulière. ComasDiaz recommande que les intervenants(es) soient particulièrement habilités à traiter de ce sujet.

Une importante source de stress pour les femmes immigrantes réside dans les conflits parfois inévitables avec leurs enfants, à mesure qu'ils s'intègrent à la société d'accueil. Les enfants sont en effet les personnes qui s'incorporent le plus rapidement et le plus facilement à la société d'adoption, et ce faisant, ils en adoptent les valeurs et modes de vie. Quelques auteures ont traité ce problème. Ainsi Barbé (1978) et Lewin (1979) mentionnent les problèmes de communication mères/ enfants, qui évoluent souvent en conflits. Outre les problèmes de communication stricte (les enfants parlant, après un certain temps, une autre langue que la mère), mère et enfants s'affrontent sur des choix de valeurs, ces derniers privilégiant souvent celles de la société d'accueil. II en résulte un isolement pour la mère, parfois la seule dans l'unité familiale maintenant nucléarisée, à maîtriser, peu ou mal la langue, et à demeurer plus près des valeurs traditionnelles de la culture d'origine.

Le cas des jeunes filles italiennes de Montréal rapporté par Bonato (1983) est intéressant à cet effet. Attachées à certaines valeurs traditionnelles mais aussi attirées par les nouvelles valeurs de la société québécoise qui leur font désirer l'égalité des rôles sociaux et la liberté de choisir leur orientation professionnelle, elles vivent une crise d'identité culturelle importante, ce qui leur fait dire : "Nous voulons briser avec un stéréotype de femme, toutefois nous refusons de croire que, pour ce faire, il faut abandonner notre culture, nous restons profondément italiennes, différentes des autres femmes ${ }^{9}$.

\section{Counseling interculturel: développement et définition}

On a commencé à souligner l'importance d'un counseling interculturel dès le début du vingtième siècle. Galton en Angleterre, Wundt en Allemagne, Hall et McKeen Cattell aux États-Unis, Binet et Henri en France ont souligné les implications, sur l'expérience des personnes, des différences individuelles selon les cultures (Herr, 1985). Leurs travaux ont permis l'élaboration de divers modèles de counseling interculturel, tant aux États-Unis qu'en Europe. Les anthropologues se sont aussi intéressés à ce sujet. Laplantine (1988) mentionne un champ 
de recherche désigné par l'expression "culture et personnalité » et qui s'est essentiellement développé aux États-Unis à partir des années 1930.

La question du counseling interculturel a toutefois été précisée à partir des années 1960. Pederson (1979), se rapportant à des études antécédentes, a signalé que les psychologues avaient été essentiellement au service de la classe moyenne, qu'il existait un problème de racisme chez les intervenants(es) et qu'il était important pour eux(elles) d'examiner leurs attitudes lorsqu'ils(elles) font face à des clients(es) d'une autre culture.

Wrenn (cité par Draguns, 1981) déjà en 1962, aux États-Unis, se faisait le porte-parole de l'imposition culturelle des valeurs et des pratiques dans la relation intervenant(e)-client(e). La conférence sur la psychologie clinique de l'American Psychological Association en 1974 (Korman, 1974) a reconnu que la connaissance de la culture d'une clientèle doit être un impératif éthique. De la même façon, on a signalé que sans une sensibilisation culturelle, le counseling ou l'intervention ne respectait pas les principes éthiques. En ce sens, l'intervention interculturelle concerne tous ceux qui travaillent auprès des gens appartenant à d'autres cultures que la culture dominante.

Les psychothérapeutes se sont aussi penchés sur les différences culturelles. Ainsi, Draguns (1975) a signalé que la culture occupe inévitablement une place prépondérante, quoiqu'ignorée, dans l'expérience de la psychothérapie. Pour leur part, Giordano et Giordano (1976) ont souligné la nécessité, pour l'intervenant(e), de maintenir un contact avec la communauté d'appartenance de ses clients(es).

Plus récemment, un auteur québécois (Saint-Amand, 1985) faisait certains constats en santé mentale. Il démontre que la conception de la maladie mentale varie considérablement selon la langue maternelle et l'origine ethnique. À son avis, "la langue maternelle constitue un vecteur essentiel de l'expression de la sensibilité ; être obligé de s'exprimer (dans une autre langue), c'est déjà s'éloigner de cette sensibilité profonde pour ne pas dire de l'essence de l'être, source de toute authenticité et donc de santé individuelle et collective ". ${ }^{10} \mathrm{St}$-Amand parle des minorités comme de groupes plus ou moins tolérés par la majorité. II remarque : "en période de crise, les contradictions sociales globales sont vécues d'une façon beaucoup plus vive par les minorités. Toutefois, en période de calme, le comportement de celles-ci est soumis à une réglementation sociale qui leur laisse moins de marge de manœuvre. Exclues du mode politique et économique, elles ont très peu de chance d'investir l'espace et de se forger une vision du monde qui leur soit propre. »11

En résumé, psychiatres, psychologues et anthropologues rappellent aux intervenants(es) en santé mentale de considérer la manière dont 
sont vécus et pensés l'origine d'une maladie et le traitement correspondant, en tenant compte non seulement des connaissances de leur culture d'origine mais aussi de celles du client.

Dans notre profession, en 1917 déjà, Mary Richmond (Devore, 1985) exprimait que les «caseworkers» ignoraient les caractéristiques raciales et spécifiques des populations ethniques. En ce sens, l'approche ethnique apparaît tôt dans le développement de la pratique du travail social. Néanmoins, ce n'est qu'au cours des années 1980 que ce champ d'intervention a pris de l'ampleur.

L'ouvrage de Devore et Schlesinger (1981) contribue de façon importante à la sensibilisation ethnique des travailleurs sociaux. II souligne les prémisses et principes suivants concernant une pratique interculturelle.

\section{Prémisses}

1. L'impact de l'histoire individuelle et collective sur les problèmes vécus par les personnes; les facettes spécifiques de cette histoire, souvent douloureuse, demeurant très présentes dans le fonctionnement social de certaines personnes.

2. L'ethnicité, source de cohésion, d'identité, de force, peut être aussi source de tension, de lutte, de rupture, spécifiquement pour les jeunes (Bonato, 1983).

3. Le travail social, tout en mettant l'accent sur le présent, doit considérer l'histoire passée des clients et la persistance de cette histoire en termes de racisme et de discrimination toujours présents dans la société d'accueil et influençant leur vie quotidienne.

\section{Principes}

1. L'évaluation psychosociale tente de cerner la situation personnelle et familiale des clients, l'impact du racisme, de la pauvreté et de la discrimination sur les personnes et groupes qui intériorisent souvent les images négatives de la société à leur endroit ; par conséquent, l'évaluation verra à déterminer les mécanismes institutionnels qui perpétuent ce racisme et cette discrimination.

2. Les habiletés du travailleur social doivent être adaptées aux besoins et particularités spécifiques des divers groupes ethniques.

3. Il est important d'être attentif au processus qui a conduit le(la) client(e) au service social, tout spécialement au continuum menant de la consultation volontaire à la rencontre obligatoire avec l'intervenant(e). 
C'est ainsi que le counseling interculturel intéresse de plus en plus d'intervenants(es). La migration croissante de populations du sud au nord y a été un facteur déterminant, particulièrement dans le contexte américain. Cette intervention a été vue comme un moyen d'aider des clients(es) victimes d'exclusion sociale, économique et politique. Son contenu s'articule autour de l'évolution des structures socio-politicoéconomiques d'une société donnée et de l'impact de cette évolution sur les minorités culturelles.

Sur le même sujet, Green (1982) signale que le travail social peut et doit être exercé dans un sens culturellement acceptable par les clients(es) et qu'il doit contribuer à accroître leur participation et leur pouvoir.

Selon Devore (1985), une contribution importante au développement de l'approche culturelle en travail social est apparue en 1982 avec l'ouvrage de McGoldrick et al. (1982). Les auteurs y présentent dix-neuf groupes ethniques avec leurs histoires de vie, l'impact de l'immigration, leurs caractéristiques familiales, l'origine des problèmes et surtout quelques suggestions thérapeutiques. Devore mentionne certaines habiletés nécessaires à l'intervenant qui travaille auprès de cette clientèle : 1. des connaissances du comportement de l'individu dans son nouvel environnement, incluant des connaissances sur la personnalité, le cycle de vie et le fonctionnement des institutions sociales; 2 . une conscience de sa propre ethnicité et de ses influences sur sa pratique; 3 . des connaissances de l'impact de la classe sociale et de l'ethnicité sur la vie des individus.

Ici au Québec, Christensen (1986) a été une pionnière dans ce domaine, s'appliquant à identifier certaines faiblesses de l'intervention sociale. Elle a ainsi signalé les peurs qui empêchent de travailler avec certaines minorités ethniques: peur de l'inconnu que représente l'étranger; peur de se sentir inadéquat; peur d'avoir à faire face à ses préjugés, biais et stéréotypes; peur qui nous fait référer le(la) client(e) à un(e) intervenant(e) "ethnique" (c'est-à-dire appartenant lui-même (elle-même) à une minorité ethnique) ou à une "agence ethnique" (c'est-à-dire un centre de services sociaux desservant majoritairement des membres de groupes ethniques); peur d'une intervention au-delà des services à court terme ou en situation de crise.

Christensen mentionne certaines attitudes qui conditionnent l'intervention des praticiens(ennes) en travail social. Ainsi, on blâme parfois les minorités d'être de mauvais clients(es), de ne pas persister dans le processus d'aide, alors qu'on évite de regarder ses propres attitudes et croyances concernant les minorités ethniques, culturelles et raciales. On généralise alors qu'au sein même des minorités, les groupes sont différents les uns des autres. Dans les établissements et à travers les 
politiques sociales, les professionnels contribuent à véhiculer mythes et stéréotypes.

En définitive, selon Christensen (1986), le travail social a négligé, en rapport avec les minorités ethniques, de modifier sa base philosophique, de préparer ses étudiants(es) à une pratique multiculturelle et de modifier ses politiques d'agence et ses modèles de service. Il faut donc examiner et évaluer ces pratiques face à des réalités nouvelles dont on découvre maintenant l'importance.

Puisqu'il est question ici des femmes immigrantes, nous aimerions finalement mettre en relation intervention féministe et counseling interculturel.

Au niveau des prémisses de l'intervention féministe, telles qu'énoncées déjà dans un ouvrage antécédent (Corbeil et al., 1983), on peut certainement avancer qu'intervention féministe et counseling interculturel se rencontrent. Ces prémisses expriment essentiellement que les racines de l'inégalité des sexes et de l'infériorisation des femmes sont sociales et non «naturelles». Telle qu'analysée dans les écrits recensés, l'ethnicité apparaît comme un construit social. Nous partageons cette perspective et ajouterions que les bases de l'inégalité entre groupes dominants et groupes minoritaires sont également sociales et non le résultat d'une infériorité « naturelle ». L'intervention féministe, tout comme l'intervention interculturelle, propose une perspective socio-politique des problèmes. Ces deux types d'intervention visent le changement social autant que le changement individuel, elles souhaitent redonner aux femmes et aux minorités ethniques un pouvoir personnel et social. La recherche actuelle aidera toutefois à préciser et à vérifier ces notions théoriques. L'étape présente en est une de construction d'instruments qui seront utilisés plus tard, lors de contacts avec un échantillon d'intervenants(es) et de groupes communautaires de l'ensemble de la région sociosanitaire 6A, desservant le Montréal métropolitain.

Les opérations suivantes sont en cours :

- Identification auprès du Centre des Services Sociaux du Montréal Métropolitain (C.S.S.M.M. - Secteurs Migrants - Immigrants) des catégories de femmes immigrantes déjà desservies ou des problématiques sociales prioritaires rencontrées dans la pratique auprès de ces femmes.

- Rencontre de quelques intervenants(es) du réseau public œuvrant auprès des immigrantes et examen de leur perception des besoins et problèmes des femmes, de leurs expériences d'intervention et du bilan qu'ils en font, incluant leur questionnement.

- Rencontre de responsables de quelques groupes communautaires desservant les femmes immigrantes, examen de leur perception des 
besoins et problèmes de ces femmes, de leurs expériences des services dispensés par les organismes publics, bilan de ces expériences en termes de satisfactions et de critiques.

\section{Notes et références}

1 Les revues et périodiques répertoriés ont été les suivants :

En français: Service social, Le service social, Service social dans le monde, Intervention, Revue internationale d'action communautaire, Revue française de service social, Revue canadienne de santé mentale communautaire, Revue canadienne de politique sociale, Sociologie et société.

En anglais : Social Work, Social Casework, Social Policy, Social Problems, The Social Service Review, Journal of Social Policy, Journal of Social Work Education, International Social Work, The International Migration Review, Ethnic and Racial Studies, Sociology and Social Research, Studies in Social Work, Social Work Today, Social Work in Education, Journal of Social Service Research, The British Journal of Social Work, Social Work Practice, Journal of Social Work and Human Sexuality.

Bilingue, anglais/français : Le travailleur social, Revue canadienne de service social, Études ethniques au Canada.

2 La bibliographie de Resources for Feminist Research, vol. 16, no 2, mars 1987, avait pour titre: "Selected bibliography of community papers concerning immigrant women in Canada : 1975-1986 " et son auteure était Joyce Scane.

3 Voici la liste des mots-clés utilisés ; ils ont été choisis en raison de leur intérêt pour le sujet de la recherche: Women, female, immigration, social, work, social work, social service, social problem, social polity, poverty, employment, labor, mental health, illness, violence, domestic, domestic violence, cheap labor, isolation, loneliness, solitude, community, community service, economy, education.

4 SIMARD, R., "Les femmes et la santé mentale : un vrai discours de folles ", dans L. Guyon, L. Simard, L. NADeAU, Va te faire soigner, t'es malade, Montréal, Stanké, 1981.

5 BROVERMAN, I.K. et al., " Sex roles stereotypes and clinical judgments of mental health ", Journal of Counsulting and Clinical Psychology, vol. 34, no 1, 1970 : 1-7. De Beauvoir, S., Le deuxième sexe, Paris, Gallimard, 1949. Millet, K., Sexual Politics, New York, Doubleday, 1970.

6 JUTEAU-LeE, D. et B. ROBERTS, "Ethnicity and feminity : (d'après nos expériences) », Études ethniques au Canada, XIII (1), p. 3.

7 David, Hélène, Femmes et emploi. Le défi et l'égalité, Québec et Montréal, Presses de l'Université du Québec et Institut de recherche appliquée sur le travail, 1986.

8 Labelle, M., G. Turcotte, M. Kempeneers et D. Meintel, Histoire d'immigrées, Montréal, Boréal, 1987, p. 223.

9 Bonato, Marto, "Elle, moi et les autres dans les minorités au Québec", Conjoncture politique au Québec, no 4, automne 1983, p. 112. 
10 Saint-Amand, Néré, folie et oppression. L'intervenant en institution psychiatrique, Moncton, Éditions d'Acadie, 1985, p. 184-185.

11 Idem, p. 186.

\section{Bibliographie}

AgGarwal, Pramila, "Business as usual in the factory", Resources for feminist Research, vol. 16, n० 1, 1987.

ANDERSON, Joan M., "Perspectives on the health of immigrant women : A feminist analysis ", Advances in Nursing Sciences, vol. 8, n० 1, $1985:$ 61-76.

BANNERJI, Himani, "Introducing racism: Notes towards anti-racist feminism", Resources for Feminist Research, vol. 16, n 2, 1987: 10-12.

BARBÉ, G., " Pour une formation des femmes immigrantes ", Études de linguistique appliquée, vol. 30, $1978: 81-91$.

Bıoom, Florence Teicher, "Struggling and surviving: The life style of European immigrant breadwinning mothers in American industrial cities 1900-1930", Women's Studies International Forum, vol. 8, no 6, 1985 : 609-620.

Bonato, Marto, "Elle, moi et les autres dans les minorités au Québec", Conjoncture politique au Québec, no 4, 1983 : 113-117.

Christensen, C.P., "Cross-cultural social work: Fallacies, fears and failings", Intervention, no 74, $1986: 6-15$.

COHEN, Rina, "The work conditions of immigrant women live in domestics : racism, sexual abuse and invisibility", Resources for Feminist Research, vol. 16, no 1, 1987: 36-38.

COMAS-DiAZ, Lillian, "Content themes in group treatment with Puerto Rican woman, Social Work with Groups, vol. 7, no 3, 1984 : 75-84.

Corbeil, C., G. LeGault, A. PÂquet-Deehy, et C. Lazure, L'Intervention féministe, l'alternative des femmes au sexisme en thérapie, Montréal, Albert SaintMartin, 1983.

DE ANDA, Diane, "Informal support networks of hispanic mothers: A comparison across age groups", Journal of Social Service Research, vol. 7, n० 3, 1984 : 89-105.

DENIS, Ann B., "Femmes: ethnie et occupation au Québec et en Ontario, 1931-1971", Etudes ethniques au Canada, vol. XIII, n० 1, 1981 : 75-90.

DENIS, Ann B., "Adaptation to multiple suhordination? Women in the vertical mosaic", Études ethniques au Canada, vol. XVIII, no 3, $1986: 61-74$.

DeVOre, W. et E.G. SCHLESINGER, Ethnic-Sensitive Social Work Practice, Saint-Louis (Mis.) C.V. Mosby Co., 1981.

DeVORE, Wynetta, "Developing ethnic sensivity for the counseling process : A social-work perspective", dans: P. PEDERSEN (éd.), Handbook of CrossCultural Counseling and Therapy, Westport (Conn.) / Londres, Greenwood Press, 1985 : 93-98.

DOUGLAS, Debbie, "Young black women speak ! ", Resources for Feminist Research, vol. $16, n^{\circ} 1,1987: 17-18$. 
Draguns, J.G., "Resocialization into culture: The complexities of taking a worldwide view of psychotherapy", dans: R.W. BRISLIN, S. BOCHNER et W.J. LonNer (éds.), Cross-Cultural Perspectives on Learning, New York, John Wiley and Sons, $1975:$ 273-289.

DRAGUNS, J.G., "Cross-cultural counseling and psychotherapy: History, issues, current status", Anthony J. Marsella et Paul B. Pedersen (éds.), CrossCultural Counseling and Psychotherapy, New York, Pergamon Press, $1981: 3-27$.

Earty, Mary Two Axe, Zonia Keyman et Helen Potrebenko, "Ethnicity and feminity as determinants of life experience ", Études ethniques au Canada, vol. XIII, no 1, $1981: 37-42$.

Engelsmann, F., H.B.M. Murphy, R. Prince, M. Leduc et H. Demers, "Variations in responses to a symptom check-list by age, sex, income, residence and ethnicity ", Social Psychiatry, vol. 7, no 1, 1972: 150-156.

EStABle, Alma, "Immigration policy and regulations", Resources for Feminist Research, vol. 16, no 1, 1987, p. 28.

FRAGNIÈRE, J.P. et K. LEY, " Des femmes immigrées s'organisent ”, Revue internationale d'action communautaire, vol. 45, no 5, 1981 : 74-81.

GIORDANO, J.S. et G.P. GIORDANO, "Ethnicity and community mental health", Community Mental Health Review, New York, Haworth Press, vol. 1, n० 3, 1976 : 4-14.

Green, James W., Cultural Awareness in the Human Services, Englewood Cliffs (N.J.), Prentice-Hall, 1982.

Guillaumain, C., "Pratique du pouvoir et idée de nature, II. Le discours de la nature", Questions féministes, vol. 3, 1978: 5-28.

GuPTA, Tania Das, "Unravelling the web of history", Resources for Feminist Research, vol. 16, no 1, 1987.

HANSON, Wynne, "The urban Indian woman and her family", Social Casework, vol. $61, n^{\circ} 8,1980: 476-483$.

HERR, Edwin L., "International approaches to career counseling and guidance ", dans : P. Pedersen (éd.), Handbook of Cross-Cultural Counseling and Therapy, Westport (Conn.) /Londres, Greenwood Press, 1985 : 3-9.

INDRA, Doreen, "The invisible mosaic: Women, ethnicity and the Vancouver Press 1905-1976", Études ethniques au Canada, vol. XIII, no 1, 1981:63-73.

JAMIESON, Kathleen, "Sisters under the skin : An exploration of the implications of feminist-materialist perspective research ", Études ethniques au Canada, vol. XIII, no 1, 1981 : 130-143.

Juteau Lee, Danielle et Barbara Roberts, "Ethnicity and feminity: (d'après nos expériences) ", Études ethniques au Canada, vol. XIII, no 1, 1981 : 1-23.

KORMAN, M., "National conference on levels and patterns of professional training in psychology : Major themes ", American Psychologist, Oxford University Press, vol. 29, $n^{\circ} 6,1974: 441-449$.

Labelle, M., D. Meintel, G. Turcotte et M. Kempeneers, "La nouvelle double journée de travail des femmes immigrantes au Québec ", Revue internationale d'action communautaire, 14/54, 1985 : 33-45.

labelle, M., D. Meintel, G. Turcotte et M. Kempeneers, "Étude comparée de la condition des ouvrières immigrées à Montréal. Présentation d'une recherche 
menée à l'UQUAM (1981-1985) ", Resources for Feminist Research, vol. 16, no 1, $1987:$ 34-35.

Labelle, M., G. Turcotte, M. Kempeneers et D. Meintel, Histoire d'immigrées, Montréal, Boréal, 1987.

LABELle, M., "Multiples visages. Les femmes immigrées au Québec ", à paraître dans: Bulletin international d'études féministes, Aix-en-Provence, automne 1988.

LAMOTHE, A., "Les autres Québécoises : étude sur les femmes immigrées et leur intégration au marché du travail québécois", Québec, Ministère des communautés culturelles et de l'immigration, 1985.

LAPLANTINE, François, L'ethnopsychiatrie, Paris, Presses Universitaires de France, 1988.

LEWIN, Ellen, "The nobility of suffering: Illness and misfortune among Latin American immigrant women ", Anthropological Quarterly, vol. 52, no 3, $1979:$ 152-158.

Lovel., Madeline C., Thuango Tran et Chi D. NGuYeN, "Refugee women : Lives in transition ", International Social Work, vol. 30, no 4, 1987 : 317-325.

MCGoldrick, M., J.K. Pearce et J. Giordano, Ethnicity and Family Therapy, New York, Guildford Press, 1982.

MCMULLEN, Lorraine, " Ethnicity and feminity : Double jeopardy ",Etudes ethniques au Canada, vol. XIII, no 1, 1981: 52-62.

MAY, Yee, "Out of silence : Voices of Chineses canadian women ", Resources for Feminist Resarch, vol. 16, no 1, $1987: 15-18$.

Neal, R. et V. Neale, "As long as you know how to do housework : PortugueseCanadian women and the Office Cleaning Industry in Toronto ", Resources for Feminist Research, vol. 16, no 1, 1987: 39-41.

NG, Roxana, "Constituting ethnic phenomenon : An account from the perspective of immigrant women", Études ethniques au Canada, vol. XIII, no 1 , $1981: 97-108$.

NG, Roxana, "Immigrant women in Canada : A socially constructed category ", Resources for Feminist Research, vol. 15, 1986 : 13-15.

NG, Roxana et Alma Estable, "Immigrant women in the labour force: An overview of present knowledge and research gaps ", Resources for Feminist Research, vol. 16, no 1, 1987:29-33.

PAREDES, Milagros, "Immigrant women and second-language education : A study of unequal access to linguistic resources ", Resources for Feminist Research, vol. 16, no 1, $1987: 23-27$.

Pedersen, Paul, "The field of intercultural counseling ", dans: P. Pedersen, W.G. Lonner et J.G. Draguns, (éds.) Counseling Across Cultures, Honolulu, University Press of Hawai, 1978 : 17-41.

Pederson, Paul, "Intercultural criteria for mental-health training ", Handbook of Cross-Cultural Counseling and Therapy, Westport (Conn.)/Londres, Greenwook Press, 1985 : 315-321.

Proulx, Michèle, Manuel de l'intervention auprès des femmes immigrantes. Centre des femmes de Montréal, Montréal, Éditions Communiqu'Elles, 1984. 
Ramkhalawansingh, Ceta, "Langage and employment training for immigrant women ", Études ethniques au Canada, vol. XIII, no 1, 1981: 91-96.

Romero-CACHINERo, M. Carmen, "Hispanic women in Canada : A framework for analysis ", Resources for Feminist Research, vol. 16, no 1, 1987 : 19-20.

RrtinA, Nancy F., "The economic status of migrant wives: An application of discriminant analysis", Sociology and Social Research, vol. 65, no 2, 1981 : 142-152.

SCHWAB, Reiko et Edward SCHWAB, "A cross-cultural comparaison of marital roles", Sociology and Social Research, vol. 65, n० 3, 1981 : 332-339.

SEWARD, J.B. et Kathryn MCDADE, "Les immigrantes au Canada: orientations souhaitables", Ottawa, Conseil consultatif canadien sur la situation de la femme, janvier 1988.

SAINT-AMAND, Néré, folie et oppression. L'internement en institution psychiatrique, Moncton, Éditions d'Acadie, 1985.

Stasiulis, Daiva K., "Rainbow feminism: Perspectives on minority women in Canada ", Resources for Feminist Research, vol. 16, no 1, 1987: 5-9.

Wolf Dresp, Christine S., "Nervios as a culture-bound syndrome among Puerto Rican women", Smith College Studies in Social Work, vol. 55, no 2, 1985 : 115-136.

ZIEBA, Mietka et Nancy HOHN, "Socio-demographic profile of immigrant women in Alberta ", Resources for Feminist Research, vol. 16, no 2, 1987: 21-22. 